\title{
DAS ADELSBERGER AHD. VATERUNSER.
}

Archivalische studien über die ortsgeschichte von Seifriedsburg in Unterfranken, auf die mich eine untersuchung der dort localisierten sage vom Hürnen Seyfried führte (s. Archiv des hist. vereins für Unterfranken und Aschaffenburg, bd. 59, 1917, s. 1 ff.), veranlaßten mich zu einer durchsicht der hausarchivalien des schlosses Adelsberg. Für die freundliche erlaubnis zu ihrer benutzung habe ich her'n rittergutsbesitzer Claus, für die gefällige übersendung des zurzeit leihweise in München erliegenden teiles herrn prof. dr. F. Mader am kgl. generalconservatorium der kunstdenkmale und altertümer Bayerns auch an dieser stelle meinen verbindlichsten dank auszusprechen. Bei dieser gelegenheit stieß ich (im januar 1917) auf die abschrift eines althochdeutschen vaterunsers, die uns zwar keine neue version kennen lehrt, aber doch mitteilung und untersuchung des textes auf seine herkunft verdient, und als denkmal der frühzeit antiquarischer interessen am althochdeutschen eines historischen zeugniswertes nicht entbehrt. Für einigè (im text bezeichnete) gütige auskünfte und hinweise bin ich herrn geheimen rat E. v. Steinmeyer, für zwei collationen (s. ur. 9) der freundlichkeit W.Streitbergs zu besonderem danke verpfliehtet.

\section{[1. Adelsberg.] Schloß und dörfchen Adelsberg liegen} etwa $30 \mathrm{~km}$ nörḍlich von Würzburg am zusammenfluß der Wern und des Mains, auf dem berghang gegenüber dem dorfe Wernfeld (anno 1158 Wernifelt, Regesta Boica I s. 235). Das schloß, ein ländliches herrenhaus, ist $1751-52$ von general Carl Reinhard Freiherrn von Drachsdorff, der das rittergut 1750 angekauft hatte, an stelle einer niedergerissenen alten burg erbaut worden. Nach der chronistischen haustradition soll diese burg im jahre 1008 von .zwei aus Hessen gekommenen brïdern Adolph und Reinhard begründet worden sein, die 1028 - 31 auch die Hohenburg (alt auch Hohenberg, jetzt Ruine Homburg a.d. Wern, etwa eine stunde östlich von Adelsberg) erbauten. Doch beruhen diese angaben nur auf späten (jetzt verlorenen) lapidarinschriften, die keinen historischen beweiswert haben (s. meine o. a. studie nr. 27-29). Als älteste 
besitzer erscheinen (im anfang des 13. jh.'s) die ritter von Hohenburg (nicht zu verwechseln mit den besitzern von Homburg = Hohenburg am Main); die Adelsberger linie starb im mannsstamm mit Jobst v. Hohenburg aus, dessen testament vom jalire 1221 datiert ist, die Hohenburger linie erlosch gegen 1381/83 (Arch. d. H. V. f. Ufr. VI, Heft 2, 92). Der besitz von Adelsberg wechselte bis in die neueste zeit so häufig, daß sich aus diesen verhältnissen keine schlüsse auf die herkunft des textes ziehen lassen. Außer der alten burg gab es in Adelsberg noch eine zweite, die sog. Diemarsburg (jetzt ebenfalls verschwunden), in der die familie von Diemar (ausgestorben 1632) hauste, die den burgplatz von Jobst v. Hohenburg erhalten haben soll (Stelzner s. 107).

Über die geschichte von Adelsberg und Hohenburg liegt keine neuere fachwissenschaftliche untersuchung vor; an älterer monographischer literatur, die jedoch für das frühmittelalter kritik vermissen läßt, sind zu nennen:

J. J. Seidner [archivar], 'Diplomatische nachrichten über den erwerb der herrschaft Hohenberg (Homburg) a.d. Wern durch das hochstift Würzburg' in der zeitschrift 'Die geöffneten archive für die geschichte des königreichs Bayern, hsg. von v. Fink', 2. jahrg., 1822/23, heft 3, s. 209-259.

Dr. F. N. Wolf [k. landgerichtsactuar], 'Geschichtliche beschreibung der burg Hohenburg ob der Wern' im 'Arch. d. H. V. f. Ufr. u. Asch.', bd. VI, heft 2, 1840, s. 83-113.

F. C. Stelzner [oberförster a.d.], 'Historische nachrichten über Gemünden und die nachbarorte' (Lohr 1888), s. 105-127 (Adelsberg).

[2. Hetterichs chronik.] Auf Adelsberg besteht ein kleines hausarchiv, das leider bei den vielen besitzwechseln sehr eingeschrumpft $\mathrm{zu}$ sein scheint, da sich verschiedene in der älteren literatur erwähnte urkunden nicht mehr vorfinden. Was noch vorhanden ist, sind fast ausschließlich jüngere archivalien und chronistisches material. Unter dem letzteren befindet sich anch cine handschriftliche 'Historisch, topographisch und statistische beschreibung des orts Adelsberg von seiner entstehung bis auf gegenwärtige zeit. Gefertigt von Joseph Hetterich, schullehrer zu A. 1843' (46 folioseiten einschließlich des titelblattes). Auf $\mathrm{s.} 1$ rechts oben steht von 
anderer hand der name 'Carl Ferdinand Werner'; aus einer anderweitigen notiz in den Adelsberger papieren ergibt sich, daß der träger dieses namens kaplan war. Auf dem rande der chronik sind von zwei verschiedenen händen notizen (in bleistift und tinte) hinzugefügt.

Über seine quellen berichtet Hetterich in der 'Vormerkung' (s. 2): 'Während meines achtjährigen wirkens als schullehrer dahier, war es stets mein regester wunsch, so viel aus alten dokumenten und der tradition kenntnis zu erlangen, um eine historische, topographische und statistische beschreibung des orts Adelsberg mit seiner umgebung fertigen zu können; allein immer war mein wille beschränkt, um den ursprung des ortes erwähnen zu können. Durch den gegenwärtigen besitzer des rittergutes, herrn Appel aus Hessenkassel, wurden mir die aufbewahrten alten akten zur einsicht und notiznahme mitgetheilt. Mit vieler mühe und großem zeitaufwand suchte ich aus den bereits unleserlichen alten notizen das interessante heraus, setzte sie zusammen, wie im nachgehenden ersichtlich ist.' Am schlusse der hs. (mit dem datum 'A., im monate april 1843') bemerkt $H$. noch: 'Beweisstellen archivalische notizen des herrn rittergutsbesitzers Heinrich Georg Appel und gemeinde-bücher.'

Unter den Adelsberger papieren befinden sich noch einige blätter älterer chronistischer aufzeichnungen aus dem 18. jh., auf deren umschlag von jüngerer hand mit bleistift notiert ist: 'Eine chronick die mit gen.-ltn. Mehling schließt, dann von herrn v. Drachsdorf fortgesetzt wurde, der aber selbst eine neue chronik anfertigen lies, die verloren gegangen.'

Solche chronistische aufzeichnungen dürften die hauptquelle Hetterichs gewesen sein (ein eingefügtes blatt zeigt auch eine andere, etwas ältere hand); auch Wolfs aufsatz hat er gekannt, und ihm bona fide allerlei pompöse floskeln entlehnt. Alte originalurkunden standen ihm wohl nur mehr spärlich zu gebote, und es ist fraglich, ob er sie "überhaupt zu lesen verstand; sein dürftiger stil und seine darstellungsart zeugen von einer sehr begrenzten literarischen bildung.

[3. Das gebet.] Wie groß oder gering Hetterichs verdienste um übermittlung ortsgeschichtlicher notizen sein mögen, 
éinen dienst hat er, ohne seine bedeutung zu kennen, der wissenschaft erwiesen, indem er aus den ihm vorliegenden archivalien ein ahd. vaterunser abschrieb und seiner chronik einverleibte.

Im anschluß an mitteilungen über das testament Jobsts v. Hohenburg anno 1221, das Hetterich als 'vor mir liegend' bezeichnet, heißt es auf s. 11: 'Ein unter den akten befindliches gebet des herrn (vater unser) hat vermuthlich der gottesfürchtige Jobst von Hohenburg hinterlassen. Es lautet': [folgt der text, s. nr. 5]. Auf dem rande neben dem gebettext steht, wie es scheint von Hetterichs hand, jedenfalls mit gleicher tinte geschrieben, die bemerkung: 'Ist mit lateinischen buchstaben zu schreiben' (H. schreibt deutsche schrift), und darunter von anderer hand mit bleistift: 'ungenau: ist gothisch!' Das 'ungenau' beweist, daß der anonymus mit 'gothisch' die schrift der vorlage, nicht die sprache des gebetes im auge hat. Hetterichs vorlage war also auch nach der niederschrift der chronik noch vorhanden, findet sich aber leider nicht mehr unter den Adelsberger archivalien; auch das testament, das Wolf 1840 ebenfalls als 'vor mir liegend' bezeichnet, ist seitdem verschwunden.

Im testament - nach den citaten bei Wolf und Hetterich, die spätmhd. dialektisch gefärbte sprachformen zeigen, ein übersetztes transsumt aus späterer zeit - kann das gebet nicht gestanden haben, da $\mathrm{H}$. sonst nicht sagen könnte 'ein unter den alten befindliches gebet'. Der ausdruck deutet auch nicht darauf hin, daß $\mathrm{H}$. das gebet aus einer älteren chronistischen aufzeichnung abgeschrieben hätte; dies ist auch dadurch ausgeschlossen, daß $\mathrm{H}$. bei seinen mitteilungen über das testament sich offensichtlich an Wolfs aufsatz vom jahre 1840 anlehnt ${ }^{1}$ )

1) Beweisend ist neben wörtlicher übernahme von floskeln der umstand, daß $\mathrm{H}$. die gleichen citate aus dem testament bringt wie Wolf, mit kleinen orthographischen abweichungen, die teils achtlose modernisierungen sind, mitunter aber doch den eindruck machen, da $B$ H. das original nachgesehen habe (mehrere $-e$, wo W. $-e n$ bietet, d. h. $n$-striche des originals aufgelöst oder ergänzt zu haben scheint); auch berichtet H. manches nus dem testament, was uber Wolfs angaben hinauageht. Dic schwicrigkeit, das testament $z u$ entziffern, erk!ürt wohl am einfachsten, weshalb H. dio gleichen citate bringt wie Wolf, der ihm als dr. Jur. gerade hierin eino besondere autoritat sein mußto. Jedenfalls besteht zum mindeston kein

Beiträge zus geschichte der deutschen sprache. 4. 
und aus einer floskel Wolfs schlüsse auf die herkunft des gebetes zog (s. u.). Man darf daher mit größter wahrscheinlichkeit schließen, daß es sich um ein loses blatt handelte, und zwar (s. o.) in 'gothischer' schrift.

Hetterichs ausdruck, das gebet habe 'vermuthlich der gottcsfïrchtige J. v. H. hinterlassen', gibt einen fingerzeig, wie er zu dieser vermutung kam. Bei Wolf fand er die wendung von dem 'testamente, in welchem sich der altdeutsche gottesfïrchtige sinn ausspricht' $(\mathrm{H}$. wiederholt dies wörtlich in seiner chronik); daher wohl der schluß, dieses für ihn jedenfalls 'altdeutsche' gebet möge von dem gottesfürchtigen Jobst hinterlassen sein. Für den abstand der sprachformen des transsumtes von denen des gebetes hatte $H$. schwerlich verständnis. Vielleicht kam dazu, daß er das blatt in einem archivbündel hinter dem testamente eingereiht fand (vgl. auch nr. 16).

[4. Formales zu H.'s mscr.] Eine sachlich bedeutungslose formalfrage der überlieferung darf gleichwohl nicht unbesprochen bleiben, da sie ohne klärung beirrend wirken könnte. Die mitteilungen über das testament beginnen auf s. 8 unten und schließen auf s. 9 mit einem citat. Darauf folgt, in gewöhnlichem zeilenabstand, also nicht etwa nachträglich eingeklemmt, die zeile:

\section{|: Nun folgt das Gebeth. :| auf der andern Seite $\Phi$}

Dann läuft der chroniktext (obne bezug auf das gebet), eingeleitet von einem zweiten verweiszeichen [hier mit * wiedergegeben], fort bis gegen schluß von s. 10, wo er mit einem unvollendeten satze und dem verweise 'p. p. $\times \S 6$ ' (d. h. fortsetzung s. $\S 6$ bei dem zeichen $X$ ) abbricht; ein seitenrest frei. S. 11 beginnt oben mitten in einem satze, der schon s. 9 im coutext (jedoch nicht zu beginn der seite) steht, und fährt dann mit dem gleichen text bis zum schlusse des letzten testamentcitats fort (gleichlautend mit dem passus auf s. 9): dieses ganze textstück auf. s. 11 ist mit der gleichen tinte wie Hetterichs handschrift durchstrichen. Darauf folgt, eingeleitet mit dem zeichen $\Phi$, der oben mitgeteilte passus ('Ein unter den akten befindliches ... es lautet') und der text des gebetes; abgeschlossen mit dem zeichen *. Die nächste zeile bringt die überschrift ' $\S 6$ ', die folgende, mit dem zeichen $\times$ eingeleitet, beginnt einen. neuen abschnitt des chroniktextes, der inhaltlich den unvollendeten schlubsatz von s. 10 wieder aufnimmt und auf s. 12 weiterläuft.

S. 11/12 war also (von Hetterich selbst) früher geschrieben als s. 9/10; Hetterich strich den anfang aus irgend einem grunde durch, verwendete

grund, Hetterichs angabe, daß ihm das testament 1843 noch vorlag, zu bezweifeln, selbst wenn er sich bei seinen mitteilungen im wesentlichen lieber an den bequemen drucktext als an das schwer leserliche transsumt hielt. 
aber das blatt, um.es nicht ganz abschreiben zu müssen, weiter, indem er durch verweiszeichen den zusammenhang sicherte. Als er den auf s.11 durchstrichenen passus auf s. 9 in neuer niederschrift wiederholt hatte, fügte er hier den hinweis 'Nun folgt das Gebeth (etc.)' an, und setzte dann seinen chroniktext fort, bis er den anschluß an $\$ 6$ erreicht hatte. Zur sicherung der seitenfolge bezeichnete er das blatt 11/12 recto oben mit der zahl 5, womit er blatt 5 (das titelblatt nicht mitgezählt) meint; sonst paginiert er nicht.

Dies wird auch durch die lage der blätter bestätigt. Die hs. beginnt mit einem quinio, von dem blatt 2 und 3 ohne textrerlust im jetzigen zusammenhange ausgeschnitten sind; sie waren jedoch beschrieben, wie schriftreste auf den schmalen, zum heften verwendeten verticalen blattresten dartun. Der vorliegende text verteilt sich auf den ursprünglichen quinio folgendermaßen: bl. 1 (s. 1/2) recto titel, verso 'Vormerkung'; bl. 2 und 3 ausgeschnitten - die rückblätter der bogen bilden bl. 9 (jetzt s. 11/12 statt 17/18) und bl. 8 (jetzt s. 13/14 statt 15/16) des ursprünglichen quinio. Bl. 4-7 (jetzt s. $3-10$ statt $7 / 8-13 / 14$ ), die den beginn des chroniktextes tragen, sind somit später geschrieben, um den text der weggeschnittenen zwei blätter zu ersetzen, der auf s. 11 noch mit dem durchstrichenen passus fortläuft.

Daraus ergibt sich, daß der hinweis 'Nun folgt das Gebeth' sich nicht auf das vorher citierte testament beziehen kann, sondern nur eine directive für den leser ist.

[5. Text.] Der folgende abdruck des gebettextes ist zeilengetreu, Hetterichs deutsche schrift jedoch in antiqua umgesetzt; zu beginn steht ein hier nicht wiederholtes anführungszeichen ["], das am schlusse durch übersehen fehlt.

Fader unser, thu in himilon bist, gewiht si nomo thin. Werde willo thin, sama se ${ }^{1}$ in himile endi in Ertha ${ }^{2}$. Unser ${ }^{3}$ tagelicha Brod gib uns hiuto, unde unsere Seulde belaz uns also auch wir belazzend unsern Seuldigen. Und in chorunga ${ }^{4}$ nidlei test du unsi. Na belase unsi fome ${ }^{5}$ Übille. $^{6}$

1 Spatium zwischen sama se etwas kleiner als normal, jedoch klar.

2 - $a$ durch aufbiegen des schlußstriches so gut wie sicher; schwerlich - $c$; $-u$ ausgeschlossen, da $\mathrm{H}$. im ganzen text (und auch sonst) bogen uber -u- setzt.

3 -r nur flüchtig angedeutet, doch sicher.

4 Vielleicht ihorunga.

5 Das ganze wort in lat. buchstaben, -e etwas größer als normal.

6 Initiale lateinisch, entweder etwas kleinere majuskel oder (minder wahrscheinlich) etwas gröBere minuskel als normal; die zwei striche (punkte) uber dem $U$.ganz klar. 
[6. Textquellen.] Der vergleich mit den fünf ahd. prosaversionen, die auf uns gekommen sind (St. Gallen, Weißenburg i. Elsa B, Bayerisches vaterunser [München-Freisinger und München-Emmeramer hs.], 'Tatian, Notker), ergibt ein merkwürdiges resultat: die erste hälfte des Adelsberger textes [Ad.] entspricht der Weißenburger version $[W]$, die zweite der Notkers [N]. Da die ahd. versionen alle bequem zugänglich sind, bedarf es zur negativen gegenprobe nicht des abdruckes sämtlicher texte und genügt die gegenüberstellung der positiven entsprechungen.

[7. Vergleich mit W.] W, dessen einzige hs. aus den 'ersten jahrzehnten des 9. jh.'s' stammt (Steinmeyer) - die abfassung setzt Kögel, Ltz. I' ${ }^{2}$ s. 457 mit Scherer '789 oder wenig später' an - ist nach E. v. Steinmeyers Kleineren ahd. sprachdenkmälern (1916) s. 29 ff. citiert; da es den gebettext zweimal bietet, zuerst zusammenhängend, dann satzweise in der auslegung, sind die abweichenden schreibungen der wiederholung $[\mathrm{W} \beta]$ hier an zweiter stelle in klammern mitverzeichnet. Differenzen zwischen $A d$. und $W$ sind durch cursivdruck hervorgehoben.

Fader unser, thu in himilon bist, gewiht si nomo thin.

Fater unser, thu in himilom bist, giuuihit si namo thin. quaeme (Qu)

richi thin.

Werde willo thin, sama se in himile endi in Ertha. uuerdhe (Uu) uuilleo (-llo) thin, sama so in himile endi in erthu.

[8. Überlieferung von Notkers VU.]. Notkers vaterunser (satzweise lateinisch und deutsch mit katechetischer auslegung) liegt in mehrfacher überlieferung vor (vgl. MSD II ${ }^{3}$ .s. 402 f.; Pipers Notkerausgabe I s. XCV ff., II s. XIV f., III s. 1; Kelle, Die St. Galler deutschen schriften, Abh. d. k. bayr. acad. d. wiss. 1890, phil.-hist. klasse bd. 18, s. 205 ff. [= Kelle $\left.{ }^{1}\right]$ und Ltg. I s. 237 ff., 398 ff. [ $=$ Kelle $\left.^{2}\right]$ ):

1. St. Galler hs. 21 des 12. jh.'s [MSD 'A', Piper 'R'].

A n m. Schilters ausgabe (Notkeri Psalterium, Ulmae 1726) wurde von Kelle und anderen als abdruck einer jetzt verlorenen hs. angesehen [MSD ' $B$ '; Kögel, Ltg. ' $R *$ '], die nach MSD entweder das original von 'A' war oder mit ihm aus der gleichen vorlage stammte. 'Die hs. wurde 1675 für De la Loubère abgeschrieben, Schilters vorlage war eine 1697 genommene copie dieser abschrift. Eine für Rostgaard 1697 angefertigte 
copie von Schilters vorlage, von Rostgaard nachträglich mit Loubères abschrift collationiert, erliegt in der $\mathrm{k}$. bibliothek in Kopenhagen (vgl. Kelle ${ }^{2}$ s. $240 \mathrm{ff}$.). Indes hat E. von Steinmeyer Beitr. 33 (1908) s. $61 \mathrm{ff}$. nachgewiesen, daß die Loubèresche abschrift nur aus dem Cod. Sangallensis 21 stammen kann. Schilters abweichungen kommt daher nur der rang von druckvarianten $\mathbf{z u}$.

2. Vadians abschrift (deutscher gebettext ohne auslegung) 1547 aus einer seitdem verlorenen hs. [MSD ' $\mathrm{V}$ ', Piper ' $S$ ']. Vadians text ist mehrfach überliefert (s. Kelle a. a. o.):

a) Im handschriftlichen nachlaß Vadians, St. Galler stadtbibliothek, und zwar doppelt - im Cod. 48 (Farrago) 1547 und im Cod. 45 (Mönchsstand) 1547, in beiden 'buchstäblich gleichlautend' (Kelle ${ }^{1}$ s. 227, Kelle ${ }^{2}$ š. 398); berichtigungen zu dem abdruck in J. v. Watts deutschen hist. schriften hsg. von Götzinger I 1875 (s. 53 ff.) verzeichnet Kelle ${ }^{1}$ s. 227 anm. 4, die abweichungen Vadians von Schobinger-Goldast [ $\left.\mathrm{N}^{2} \mathrm{c}\right]$ s. 228 anm. 4.

b) In Joh. Stumpffs chronik (Zürich 1548) bl. 295 b nach Vadians mitteilung; abdruck bei Piper II s. XIV.

Aus Stumpff wiederholt in Gesners Mithridates 1555, bei Nathan Chytræus, und in Kirchhoffs Wendunmut (MSD). Kelles angabe (Ltg. I s. 399) 'auch Gesner erhielt [den text] von Vadian' ist ein versehen, denn Gesners text stimmt (bis auf wesenlose kleinigkeiten) vollständig mit Stumpff, auch wo dieser allein steht, so in den druckfehlern 'hinto', ' $\ddot{u} n s e r$ ', 'üns' (bei G. je einmal; bei Stumpff durch verwendung einer gotischen type anderer größe mitten in der sonstigen antiquaschrift als druckfehler kenntlich).

c) In M. Goldasts Alam. Rerum Scriptores III (1606) s. 47 (im abdruck von Vadians Farrago). Goldasts vorlage war eine abschrift von Vadians Cod. 48 durch Schobinger (Kelle ${ }^{1}$ s. 224), der 'den wortlaut des Pater noster mit der hs. verglichen hat': s. Kelle ${ }^{1}$ s. 227 f., wo nachgewiesen wird, daß Vadians eigene excerpte durch auslassungen, nachweisliche änderungen und durch das fortlassen der accente dem SchobingerGoldast-text an genauigkeit nachstehen. Schobingers mscr. ist noch erhalten, vergleichung mit Goldast und mit Vadians concept bei Kelle ${ }^{1}$ s. 228 anm. 4; abdruck von Goldasts text (nach der 2. ausgabe von 1661) bei Piper II s. XV.

3. Einige citate aus einer verlorenen Notker-hs. - nach Kelle identisch mit der von Vadian benutzten - bietet M. Freher 1609, s. Kelle² s. 400 mit abdruck. 
4. Cod. lat. 7637 Indersdorf. 237 (München), 12.jh., fol. 45 (MSD 'I', Piper 'T'); abdruck (nicht völlig zuverlässig) bei Maßmann, Die deutschen abschwörungs-(etc.)formeln (1839) nr. 56 (und gebettext allein daraus wiederholt nr. 52); lesarten in MSD und bei Piper II s. XLVIII).

5. Bayerische umarbeitung in der Ambras-Wiener hs. 2681 (wahrscheinlich aus Wessobrunn), 11.jh. (MSD 'Catechismus B', Piper ' $Y$ ').

Die sonstigen bruchstücke von handschriften des Psalters (usw.) kommen für das vaterunser nicht in betracht.

[9. Vergleich mit $\mathrm{N}^{1-5}$.]

Die texte $1-5$ sind im folgenden mit $N^{1}$ usw. bezeichnet. Citiert sind $N^{1}$ und $N^{5}$ nach Piper (mit einsetzung der hs. lesart, wo Piper geändert hat), $N^{2} b$ (Stumpff) und die druckabweichungen Schilters [Sch] von $\mathrm{N}^{1}$ nach den originalen, $\mathrm{N}^{2} \mathrm{c}$ (Goldast 1606) und $\mathrm{N}^{4}$ (Cod. Ind.) nach abschriften der originale, die ich der gefälligkeit W. Streitbergs verdanke, $\mathrm{N}^{3}$ (Freher) nach Kelles Ltg. I, 400 (mit kleinen correcturen [V, f, ó] nach mitteilung E. v. Steinmeyers), die lesarten aus Vadian $\left(\mathrm{N}^{2} a\right)$ nach Kelle ${ }^{1}$ s. 228 anm. 4 (wo vermutlich $\int$ und $s$ wie bei seinen Frehercitaten ausgeglichen sind). Die abweichenden lesarten von Sch sind als anm. zu N ${ }^{1}$ verzeichnet (ohne berücksichtigung der differenzen von $\int$ und $s$, da Pipers texte keinen unterschied machen), die von $\mathrm{N}^{2} \mathrm{~b}$ sowie Kelles angaben über die varianten Vadians als anm. zu N ${ }^{2}$ c. Bei $\mathrm{N}^{3}$ ist $\mathrm{zu}$ beachten, daB Freher nur die hier citierten wörter bietet. Lateintext und auslegung (die in $\mathrm{N}^{2}$ und Ad. fehlen) sind übergangen. Bezeichnende übereinstimmungen cursiviert.

Ad.

Unser tagelicha Brod gib uns hiuto,

$\mathrm{N}^{1}$ (St. Gall.) Vnser ${ }^{1}$ tágelicha ${ }^{2}$ brôt. ${ }^{3}$ kib uns hiûto.

$\mathrm{N}^{2} \mathrm{c}$ (Gold.) Vnfer tágolicha ${ }^{a}$ brôt kib vns hiúto.

$\mathrm{N}^{3}$ (Freh.) Vnfer tagolicha brot.

$\mathrm{N}^{4}$ (Ind.) vnfer tagelich brôt kib unf* hiuto.

$\mathrm{N}^{5}$ (Bayer.) Vnsir tagelichiz prot gib uns hiuto.

1 Sch Unfer 2 Sch durchgängig keine accente (usw.) 3 Sch kein punkt

a $\mathrm{N}^{2}$ b durchgängig keine accente (usw.) $\quad \mathrm{N}^{2} \mathrm{~b}$ hinto:

* hiuto ganz nah an unf gerückt

Ad.

$\mathrm{N}^{1}$

$\mathrm{N}^{2} \mathrm{c}$

$\mathrm{N}^{4}$

$\mathrm{N}^{5}$

4 Sch Unde

c $\mathrm{N}^{2} \mathrm{~b}$ unde

unde unsere Seulde belaz uns

Vnde ${ }^{4}$ únsere sculde belâz uns. ${ }^{5}$

$V{ }^{c}{ }^{c}$ únfere fcúlde belâz ${ }^{d}$ úns, ${ }^{e}$

Vnde vnfer fculde belaz unf.

Vnde unsere sculde belaz uns.

5 Sch unz,

d $\mathrm{N}^{2}$ a belasz (Cod. 49 beláz)

e $N^{2} b$ üns । 
Ad. also auch wir belazzend unsern Seuldigen.

$\mathrm{N}^{1}$ also ouh uuir belazen unseren sculdigen.

$\mathrm{N}^{2} \mathrm{c} \quad$ álBo ouch vvir belázend ${ }^{\mathrm{f}}$ vnferng fcúldigen.

$\mathrm{N}^{2} \mathrm{~b} \quad$ als auch wir belafend unferen fculdigen;

$\mathrm{N}^{4} \quad$ alfouch wir belazzen unferen fculdigen.

$\mathrm{N}^{5} \quad$ also ouch $\left[{ }^{*}\right]$ firlazen unseren scolaren.

f $\mathrm{N}^{2} \mathrm{a}=\mathrm{N}^{2} \mathrm{~b} \quad \mathrm{~g} \mathrm{~N}^{2} \mathrm{a}$ vnseren

* [wir] von Piper ergänzt

Ad. Und in chorunga nidlei test du unsi.

$\mathrm{N}^{1} \quad \operatorname{Vnde}^{6}$ in chorunga nelêitest ${ }^{7}$ dû $^{8}$ únsih.

$\mathrm{N}^{2} \mathrm{c} \quad V n d^{\mathrm{h}}$ in chórunga nit leítest ${ }^{\mathrm{i}} \mathrm{du}$ únfich. $\mathrm{k}$

$\mathrm{N}^{3} \quad$ Vnde in chórunga ne leitift tu vnfih.

$\mathrm{N}^{4} \quad$ Vnde in chorunge neleiteft tu unfich.

$\mathrm{N}^{5} \quad$ Vnde in dia chorunga neleitist $d u$ unsih.

6 Sch Unde 7 Sch getrennt (ne 1.) 8 so hs., Piper tû, Sch du

$h \mathrm{~N}^{2} \mathrm{~b}$ und i $\mathrm{N}^{2} \mathrm{~b}$ leiteft $k \mathrm{~N}^{2} \mathrm{~b}$ kolon

Ad. $\quad N a$ belase unsi fome Übille.

$\mathrm{N}^{1} \quad$ Nube* $^{*}$ lose unsih fone ubele. 9

$\mathrm{N}^{2} \mathrm{c} \quad N u^{1}$ belôfe ${ }^{\mathrm{m}}$ únfih $^{\mathrm{n}}$ fóme $^{\mathrm{n}}$ úbele. $^{\circ}$

$\mathrm{N}^{4} \quad$ Nube lofe unfich fone ubele.

$\mathrm{N}^{5} \quad$ Suntir irlose unsih fone demo ubile.

* so hs., Piper Núbe 9 Sch komma

I $\mathrm{N}^{2} \mathrm{a}$ und $\mathrm{b}$ Nun $\mathrm{m} \mathrm{N}^{2} \mathrm{~b}$ belofe $\quad \mathrm{N}^{2} \mathrm{a}$ und $\mathrm{b}$ unsich fone

o $\mathrm{N}^{2} \mathrm{~b}$ ubele, \&c.

Es ist auf den ersten blick ersichtlich, daß Ad. sich dem $\mathrm{N}^{2} \mathrm{c}$-text auf das engste anschließt; doch stimmt (gegen $\mathrm{N}^{2} \mathrm{c}$ ) 'tagelicha' mit $\mathrm{N}^{1}$, ' $g \mathrm{ib}$ ' und 'Übille' mit $\mathrm{N}^{5}, z z$ in 'belazzend' mit $\mathrm{N}^{4}$; die übereinstimmung ' $a u c h$ ' mit N ${ }^{2} \mathrm{~b}$ (Stumpff) gegen 'ouch' $\mathrm{N}^{2} \mathrm{c}$ scheidet von vornherein als bloßer zufall aus, da Stumpff infolge seiner vielen abweichungen (hinto, üns, als, belafend, unseren, Nun, fone) als quelle für Ad. ausgeschlossen ist. $\mathrm{Ob}$ die anderen ubereinstimmungen auf eine abweichende hs. der $\mathrm{N}^{2}$-klasse deuten oder anders $\mathrm{zu}$ erklären sind, laBt sich erst nach aufhellung einer reihe von vorfragen bestimmen (s. Mr. 12). 
[10. Die hs. N2.] Der hs. $\mathrm{N}^{2}$ (Vadians vorlage) wird von Kelle und von Kögel ein sehr hohes alter zugesprochen. Ersterer hält sie für die im jahre 1027 gefertigte abschrift des originals (Abh. s. 232), letzterer möchte in ihr 'vielleicht' das original selbst sehen (Ltg. II s. 610). Es bleibt unaufgeklärt, wie dies bei den jungen formen der uns überlieferten $\mathrm{N}^{2}$-texte möglich sein soll. Auch Bruckner weist (in der 2. auflage von Kögels Grundriß § 131) diese annahme zurück: 'Diese hohe stelle kann der hs. nicht zugekommen sein, da sie nach den proben, die Goldast und andere mitteilten, einen ziemlich fehlerhaften text aufwies.'

Nicht geringer ist die schwierigkeit, die aus Kelles annahme erwächst, Frehers citate seien aus derselben Notker-hs. geflossen, die Vadian benutzte. Die geschichte der hs., die Kelle darstellt, scheint darauf zu deuten. Auffälligerweise aber hat Freher (im bereiche der kurzen vaterunser-citate) $\alpha)$ dreimal die älteren formen gegenüber den $\mathrm{N}^{2}$-texten (Vnde für Vnd, ne für nit, un/ih für ún/ïch) und weicht $\beta$ ) żweimal $\mathrm{ab}$ ('leitift tu' für 'leitest $d \mathrm{u}$ '), d. h. von 10 wörtern sind 5 anders geschrieben. Dies läßt nur den schluß zu: entweder sind die jüngeren formen, die $\mathrm{N}^{2} \mathrm{a}$ und $\mathrm{c}$ übereinstimmend aufweisen, auf Vadians abschrift zurückzuführen, während Freher die lesart der hs. hat (wogegen aber gruppe $\beta$ spricht), oder war die hs., die Freher benutzte, nicht mit dem original der $\mathrm{N}^{2}$-texte identisch; daß Freher die älteren formen durch stillschweigende emendation hergestellt hätte, darf wohl für ausgeschlossen gelten.

Nun hat Kelle nachgewiesen, daß Schobinger den text des vaterunser (in der abschrift der Vadianschen abhandlung, die er Goldast übersandte) nochmals mit der hs. selbst verglichen und ungenauigkeiten Vadians berichtigt hat. Sollte er bei dieser collation gleich drei fälle übersehen haben, darunter eine so auffällige, in die-augen springende, abweichung wie nit für ne, während er sonst bis ins kleinste bessert und selbst accente ergänzt? Und selbst mit dieser unwahrscheinlichen annahme wären noch nicht alle jungen formen des Goldasttextes erklärt; die falsche spatiierung 'Nú belôfe' und die jungen formen 'vvir belázend' und 'fóme' bei Goldast können unmöglich auf Vadian zurückgehen, da 
Schobinger an allen drei stellen gegen Vadian (also nach der hs.) geändert hat ( $\mathrm{N}^{2}$ a liest 'Nun', 'belasend', 'fone') und die accente zufügt, also gerade diese wörter sich sehr genau angesehen hat (Stumpff $-\mathrm{N}^{2} \mathrm{~b}$ - beweist natürlich nichts, da er seinen text von Vadian erhielt).

Man muß daraus schließen, daß die jungen formen in der tat der hs. $\mathrm{N}^{2}$ entstammen und nicht auf das conto Vadians gesetzt werden können. Dann kann aber (so weit die schlüsse aus der hier untersuchten váterunserpartie reichen) weder Frehers text aus der verlorenen hs. $\mathrm{N}^{2}$ stammen, noch diese dem 11. jh. angehört haben. Die analogische. ausdehnung der endung -nt der 3. pl. ind. auf die 1. pl.. begegnet erst in mhd. zeit; sie ist nach Weinhold, Mhd. gr. ${ }^{2} \S 369$ 'in jüngerer zeit, namentlich alemannisch, oft' anzutreffen, nach Weinholds Alem. gr. § 342 'seit dem 14. jh.', mit ältestem beleg anno 1300; daselbst auch beispiele für -nd. Zur entstehung der hs. $\mathrm{N}^{2}$ im 14. jh. ( $\left.a q u o\right)$ würde auch die verständnislose spatiierung 'Nú belôfe' und 'nit' für 'ne' völlig passen.

[11. Die vorstufen von H.] Die vorlage Hetterichs läßt sich nunmehr genauer erfassen. Da Ad. keine einheitliche und selbständige version ist, sondern sich aus zwei bereits voraus existierenden originalversionen zusammensetzt, schiebt sich zwischen Hetterichs abschrift und den W- und $\mathrm{N}$-text mindestens éine zwischenstufe, die hs. des compilators (C), die nach dem alter des benutzten $\mathrm{N}^{2}$-textes nicht älter sein kann als das 14. jh. (a quo). Das würde chronologisch auch $\mathrm{zu}$ der marginalangabe über die 'gothische' schrift der vorlage Hetterichs stimmen, die, rund gesprochen, auf das 15.-16. jh. weisen könnte (vgl. auch nr. 13, majuskeln, und nr. 17, schluß).

Für die abweichungen zwischen Ad. und den überlieferten texten von $\mathrm{W}$ und $\mathrm{N}$ können daher mindestens drei quellen in frage kommen: 1 . die schreibung der von $\mathrm{C}$ benutzten hss. der originalversionen, die ja (theoretisch) mit den erhaltenen nicht identisch gewesen $\mathrm{zu}$ sein brauchen (über drucke als eventuelle vorlage von $\mathrm{C}$ s. $\mathrm{nr} .15$ und 16); 2. die schreibung von $\mathrm{C} ; 3$. versehen Hetterichs oder eventueller mittelglieder (M) der uberlieferung zwischen $\mathrm{C}$ und $\mathrm{H}$ (vgl. nr. 17). Obwohl eine positive entscheidung uber die aufteilung der abweichungen 
auf diese drei stufen nicht in allen fällen möglich ist, lassen sich doch einige punkte klären.

[12. Verlesungen.] Da Hetterich den text stellenweise nicht verstand und die ihm ungewohnte schrift der vorlage ilhn zu besonderer aufmerksamkeit nötigte, ist an flüchtigkeiten und unachtsamkeiten seiner abschrift nicht zu denken. Bei seinen citaten aus dem testament kommen solche wohl vor, aber an dem testament interessierte ihn der inhalt, nicht die sprachform, die zudem der modernen bedeutend näher steht und daher unbewußte modernisierungen begünstigte. Das gebet dagegen, das er als vaterunser erkannte, teilt er gerade wegen seiner alten, abweichenden sprachform mit: bewußte modernisierungen sind daher ausgeschlossen. Als dorfschullehrer gewohnt, die schreibhefte seiner schüler zu überwachen und corrigieren, wird er seine eigene abschrift nicht minder genau behandelt haben. Aus allen diesen gründen kommen für $\mathrm{H}$. nur verlesungen als fehlerquelle in betracht.

Als verlesungen H.'s (oder einer mittelstufe, vgl. nr. 17) sind unzweifelhaft aufzufassen: die falsche spatiierung 'nidlei test', se für sc ('Seulde', 'Seuldigen'), ' $\mathrm{N} a$ belase' (für ' $\mathrm{N} u$ belose'; die falsche spatiierung bereits in $\mathrm{N}^{2}$ ), wohl auch, da gleichartig, se für so. Auch 'nomo' und 'Ertha' (dat.) sind gewi $B$ nicht dem ahd. original von $C$ zuzuschreiben; zwar gibt es ahd. beispiele für $a>0$ vor nasalen (Braune $\S 25,1$; Franck $\S 9$ ) und für dative der $\bar{o}$-fem. auf $a$ (Braune $\S 207,5$; Franck $\S 137$, aber nur vereinzelt, und es wäre seltsam, daß der von $C$ benutzte W-text gleich zwei abnormen (dazu noch 'Fader') auf einmal aufgewiesen hätte. Zweifelhaft bleibt, ob nomo verlesung Hetterichs (bez. einer mittelstufe) ist oder schon schreibung von $\mathrm{C}$ war, da verdumpfung von $a>0$ im alemannischen, besonders seit dem 13. jh., häufig vorkommt (Weinhold, Al. gr. § 25; für obd. überhaupt vgl. Weinhold, Mhd. gr. $\S 23)$. Ebenso kann man schwanken, ob ' $a u c_{h}$ '. ( $\mathrm{N}^{2} \mathrm{c}$ ' $o u \mathrm{~h}$ ') und 'tagelicha'. ( $\mathrm{N}^{2} \mathrm{c}$ 'tagolicha') $\mathrm{H}$. (bez. einer mittelstufe) oder C zufallen; au tritt seit ende des 13. jh.'s wieder stärker hervor und nimmt an häufigkeit im 14.-15. jh. beständig zu (Weinhold; Mhd. gr. ${ }^{2} \S 1$ 125, 127); 'tagelicha' zeigt schon die hs. $N^{1}$ : beides ist als naheliegende sprachmöglichkeit daher auch schon $\mathrm{C}$ zuzutrauen. 
Jede verlesungsmöglichkeit aber ist ausgeschlossen bei den von $\mathrm{W}$ und $\mathrm{N}^{2} \mathrm{c}$ abweichenden formen gewilht (ge, synkope), gib, belazzend (mit zz), unsi (2 mal), Übille ( $\ddot{i}, i, l l)$ und den $d$ für $t$ (nid, Brod, Fader). Hetterichs abschrift scheidet als quelle für sie aus; sie sind daher C (oder zwischenstufen) zuzuweisen. Bei so vielen abnormen ist nicht daran zu denken, daB sie alle aus den vorlagen von $C$ stammen sollten; die übereinstimmung einzelner davon mit Notkerhandschriften anderer klassen (s. nr. 9) verliert daher bei der weitgehenden willkür von $\mathrm{C}$ jeden beweiswert für die theoretische möglichkeit, daß $\mathrm{C}$ eine andere hs. der $\mathrm{N}$-version benützt haben könnte, als $\mathrm{N}^{2}$ selbst oder eine abschrift davon, und sind auch als zufall erklärbar.

[13. Schlüsse auf die orthographie des compilators.] Der zweimalige gebrauch des (stimmlosen) $d$ für $t$ ('nid', 'Brod'; der dritte fall, 'belazzend', stammt aus $\mathrm{N}^{2}$ ) zeigt eine orthographische gepflogenheit von C, für die auch 'Fader' im 1. teile einen beleg bietet: beispiele für 'einzeln schon früh und später häufig' erscheinende inlautende $-d$-schreibungen für - $t$ - im alemannischen s. Weinhold, Al. gr. $\S 180$, im mitteldeutschen Mhd. gr. ${ }^{2} \S 188$; an eine abweichung der von $\mathrm{C}$ benutzten W-handschrift ist umsoweniger zu denken, als gerade im Weißenburger katechismus und im Isidorus nur 'fater' vorkommt, s. Franck, Altfrk. gr. $\S 89,2$, ebenso auch in Otfrid, s. Kelles glossar.

Auffallend ist unsi für unsih; da unsih nur 'bis ende des 13. jh.'s nachweislich ist' und 'im 13. jh. immer seltener wird' (Weinhold, Mhd. gr. ${ }^{2} \S 472$ ), war es C wohl schon völlig ungeläufig; vielleicht ist an den (besonders im md. häufigen) schwund des auslautenden postvocalischen $h(-c h)$ zu denken, der allerdings normalerweise nur nach langen vocalen eintritt (Weinhold § 242, 246; Michels, Mhd. el.-b. ${ }^{2} \S 118$ ), vielleicht auch an eine ungenauigkeit einer mittelstufe.

Die majuskeln als absichtliche änderung H.'s aufzufassen, hindert die regellosigkeit ihres gebrauchs. Hätte $\mathrm{H}$. sich bona fide berechtigt gefühlt, dem modernen brauche zu folgen, so müBte man consequenz erwarten; da aber von 10 substantiven im satzinnern nur 5 (oder 4) majuskeln zeigen, ist diese regellosigkeit entweder einer zeit zuzuschreiben, in der willkurlicher 
wechsel ubblich war (wie z. b. in der Ambraser handschrift vom anfang des 16. jh.'s, vgl. das facsimile im Reallex. d. germ. altertumskunde bd. I, tafel 28; nr. 20), oder aber könnte Hetterich zufälligen größenunterschieden der buchstaben dort, wo moderner brauch majuskel heischt, etwa diese bedeutung zugeschrieben haben (auf unsicherheit der auffassung könnte man die schreibung von 'Übille' s. anm. 6 in nr. 5 deuten); doch ist die zweite alternative wenig wahrscheinlich. Es mag nicht unerwähnt bleiben, daß die zwei textteile dabei einen (zufälligen?) unterschied aufweisen: von je 5 substantiven im satzinnern beginnen mit majuskel im $\mathrm{W}$-text 1 , im $\mathrm{N}$-text 4 (oder 3).

Die fortlassung der accente wird wohl $\mathrm{C}$ zuzuschreiben sein, der sie als etwas seiner zeit nicht mehr geläufiges über bord warf.

[14. Die von C benutzten hss.] Daß C den N-text nur aus der hs. $\mathrm{N}^{2}$ (direct oder indirect) bezogen haben kann, geht aus dem vergleiche unmittelbar hervor. Sein W-text weist einige abweichungen von der W-hs. auf, die schon ahd. denkbar sind und allenfalls auf eine andere etwas jüngere ahd. hs. von $\mathrm{W}$ deuten könnten. 1. himilon (W -om), 2. gewiht (W gi-), 3. und 4. Werde (3. majuskel: W $\alpha$ uu-, W $\beta U u-;$ 4. -d-: $\mathrm{W}-d h$ ), 5. willo (W $\alpha-e o, \beta-o$ ) [über Fader s. nr. 13, nomo, Erth $a$ s. nr. 12]. Ob einem compilator frühestens des 14. jh.'s diese änderungen zuzutrauen sind, muß subjectivem urteil überlassen bleiben. Auf 3. ist natürlich nichts zu geben, 2. und 4. entsprechen mhd. gebrauch, lagen also für $\mathrm{C}$ nahe; 1 . und 5. dagegen wären änderungen, die trotzdem noch kein mhd. wortbild herstellen; immerhin nähern sie sich dem mhd. insofern, als sie das $-m$ im dat. pl. der substantiva und die lautcombination -eo - beides der zeit von $C$ nicht mehr geläufig - vermeiden, so daß bewußte oder unbewußte angleichung an die eigene sprachform. bei $\mathrm{C}$ wohl denkbar ist.

[15. Vergleich mit drucken.] Da das 14. jh. nứ den terminus a quo für $\mathrm{C}$ bildet, $\mathrm{mu} \beta$ auch die frage aufgeworfen werden, ob $\mathrm{C}$ nicht aus drucken geschöpft haben kann. Eine compilation von $\mathrm{W}$ und $\mathrm{N}$ wie in Ad. ist bisher (wie E. von Steinmeyer mir bestätigt) nicht bekannt. Für $\mathrm{N}$ würde da Goldast (1606) am nächsten liegen; da aber'der Goldast-text, 
so viel wir wissen, sich mit der hs. $\mathrm{N}^{2}$ deckt (s. nr. 10), ist kein schluß aus der textvergleichung möglich. Man könnte höchstens anführen, daß nur in den $\mathrm{N}^{2}$-auszägen der lateinische text und die auslegung fehlt wie in Ad.; aber die absicht, den deutschen vaterunsertext für sich allein herzustellen, konnte verschiedenen individuen zu allen zeiten naheliegen (vgl. nr. 17).

Den terminus a quo für die compilation würde der erste druck von W in Eckhardts Catechesis 1713 bilden. Eckhardts text (s. 60) weicht von der hs. (in der Adelsberger partie) nur in folgenden punkten ab: 1 . W $\alpha \beta$ himilon (hs. -om); 2 . W $\alpha G i$ (hs. gi); 3. W $\alpha$ Uuerdhe (hs. uu-); 4. W $\beta$ endhi (hs. $-d$-), jedoch W $\alpha-d$ - wie die hs. Von diesen differenzen stimmt 1 und 3 (majuskel) mit Ad., nicht aber 2.; 4 kommt, da bloß in $W \beta$, nicht in betracht (Ad. $=\mathrm{W} \alpha$ und E). Ferner hat Ad. 'Werde' gegen $\mathrm{E}$ und $\mathrm{W}(-d h-)$, willo gegen $\mathrm{E}$ und $\mathrm{W} \alpha(-e o)$, und ein komma nach dem zweiten wort, das in $\mathrm{E}$ fehlt. Eckhardts W-text bietet also keine handhabe, $\mathrm{C}$ aus drucktexten abzuleiten.

Da Eckhardt 1713 auch Goldasts text abdruckt, so daß ein compilator hier $\mathrm{W}$ und $\mathrm{N}^{2}$ beisammen finden konnte, ist auch eine collation dieses textes angezeigt. Von Goldast weicht $\mathrm{E}$. (in der Adelsberger partie) nur in zwei schreibungen $\mathrm{ab}$ : 1. G. 'al $\beta 0$ ', E. 'al/o'; G. 'ouch', E. 'ouh' (Ad. 'also', 'auch'). Im 2. fall stimmt also Ad. mit G. gegen E., im 1. mit E. gegen G.; da Goldasts ' $B$ ' schwerlich aus der hs. stammt, verliert die ohnedies nichtssagende einzige übereinstimmung von Ad. mit E., der die schwerer wiegende abweichung gegenübersteht, jede beweiskraft. Die fortlassung der accente und die ersetzung der anlautenden vocalischen $v_{-}\left(V_{-}\right)$durch $u-\left(U_{-}\right)$bei $\mathrm{E}$. kehrt zwar in Ad. gleicherweise wieder, kann aber schwerlich einen zusammenhang wahrscheinlich machen, da solche modernisierungen auch bei $\mathrm{C}$ sehr nahe liegen.

Wie wenig auf vereinzelte übereinstimmungen in solchen orthographischen minutien zu geben ist, zeigen zwei druckfehler in Eckhardts $\mathrm{N}^{\mathrm{B}}$-text (s. $81 \mathrm{ff}$ ), die durch zufall mit Ad. stimmen. Es heißt bei $\mathrm{K}$.: ne lei $\|$ tift $d u$ unfi (\| zeileu und spaltenschlub, ohne bindestrich). Damit stimmt zwar das erste unsi von Ad., aber nicht das zweite (wo k. correct unfih hat); die falsche spatiierung nillei test in Ad., aber weder nid filr ne, test fur tift noch die falsche likatur nidlei. Ebenso hietet owar $k$.

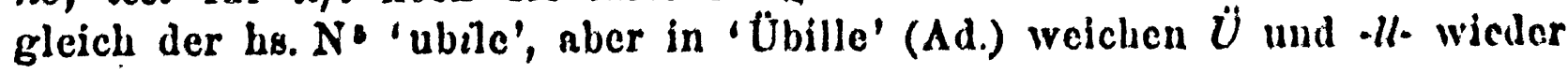


ab. Da $\mathrm{N}^{\mathrm{s}}$ iufolge seiner mehrfachen abweichungen überhaupt als quelle filr Ad. ausgeschlossen ist, müßte man, um einen zusammenhang mit $\mathbf{E}$. zu erweisen, annehmen, daß $\mathrm{C}$ aus $\mathrm{E}$. zwar $\mathrm{N}^{2}$ abschrieb, aber aus Eckhardts $\mathrm{N}^{5}$ minimale abweichungen einsetzte, ohne doch auch nur éines dieser wörter voll nach $\mathrm{N}^{5}$ zu schreiben, was höchstens zum zwecke einer raffinierten mystification überhaupt denkbar wäre, die hier ganz ausgeschlossen ist.

[16. Gründe gegen druckcompilation.] Da die untersuchung Eckhardts, der als denkbare quelle beider texte am ehesten in frage kommen konnte, negativ ausgefallen ist, dürfen nun mit größerer sicherheit, als vorher möglich gewesen wäre, die gründe formuliert werden, die von vornherein gegen eine compilation aus drucken zu sprechen scheinen. Sie könnte (wegen W) erst nach 1713 erfolgt sein.' Würden züge des 18. jh.'s den eindruck 'gothischer' schrift machen? Hätte ein blatt des 18. jh.'s so altertümlich ausgesehen, daß Hetterich - mag er noch so unkritisch gewesen sein - es auf das 13. jh. zurückführen konnte? War es nicht eher eine annähernde schriftgleichheit und altersähnlichkeit mit dem spätmhd. testamentstranssumt, was $H$. bei seinem schlusse (vgl. nr. 3) bestärkte oder leitete? Ist es wahrscheinlich, daß H. eine hs. des 18. jh.'s so oft verlas, oder daß ein compilator des 18. jh.'s, für den kein anderes interesse als ein philologisches denkbar ist, seine druckvorlagen -mit so großer orthographischer gleichgültigkeit abschrieb? Aus allen diesen gründen darf im vorhinein bezweifelt werden, daß eine vergleichung aller $\mathrm{N}$ - und $\mathrm{W}$-drucke des 16.-18. jh.'s, zu der mir zu viele ausgaben unzugänglich sind, anhaltspunkte für eine ableitung von $\mathrm{C}$ aus drucken ergeben würde.

[17. Ursprung von Ad.] Die abschrift eines ahd. vaterunsers im 15.-17. jh. würde sich am ehesten aus.dem antiquarischen interesse eines humanisten erklären. Wäre die vorlage Hetterichs ein nach Adelsberg verflattertes blatt aus dem besitze eines humanisten, so bliebe aber unverständlich, wie dieser - entgegen seinen interessen - die schreibung so nachlässig behandelte und auf den gedanken geraten konnte, zwei versionen je eine hälfte $z u$ entnehmen und die andere fallen $\mathrm{zu}$ lassen.

Ein deutsches vaterunser auf einem losen blatte könnte auch einen praktischen, seelsorgerischen zweck gehabt haben: 
man kann sich sehr wohl vorstellen, daß ein geistlicher des spätmittelalters, ein beichtvater oder schloßkaplan, es für einen lesekundigen laien niederschrieb, und die orthographische gleichgültigkeit von $\mathrm{C}$ deutet in der tat auf eine hand, die unbekümmert um philologische akribie den eigenen gepflogenheiten nachgab. In der umgebung von Adelsberg gab es mehrere klöster, denen der besitz einer W- und einer N-hs. wohl zuzutrauen wäre: die Benediktinerabteien Neustadt a. M. (8. jh. - 1803) und Aura a. d. frk. Saale (1108-1561), und, noch näher an Adelsberg, das Benediktinerpriorat Schönrain (11. jh.-1526) und das adelige Cistercienserinnenkloster Schönau (1189-1564). Schönrain war eine Hirsaụer gründung, was bei der übermittlung einer alemannischen Notkerhs. eine rolle gespielt haben könnte; ein fragment einer Notkerhs. des 12.jh.'s ist in Aschaffenburg zutage gekommen (entdeckt von A. Chroust, hsg. von E. v. Steinmeyer, Beitr. 30). Aber was konnte (ganz abgesehen von der auch in diesem falle rätselhaften compilation) einen geistlichen des spätmittelalters bewegen, für praktische privatzwecke ein ahd. vaterunser zusammenzustellen?

Über die gründe, die den compilator zur klitterung zweier texte bewogen, ist nichts zu ermitteln. Auf die ursprüngliche verwendung des vaterunsers dagegen fällt vielleicht ein licht aus der nach abschluß meiner untersuchung erschienenen notiz von Schiffmann (Beitr. 42, 1917, s. 344-45), auf die E. von Steinmeyer mich aufmerksam gemacht hat. Schiffmann weist nach, daB im kloster St. Zeno bei Reichenhall (Bayern) zwei alte marmorplatten mit dem paternoster, credo und dekalog existieren, daß nach dem zeugnis eines einblattdruckes des paternoster und credo vom anfang des 16. jh.'s diese gebete im Salzburger dom in marınor eingemeißelt waren (der dom wurde $\mathrm{zu}$ ende des 16. jh.'s niedergerissen), und dab ein vorläufer des Linzer druckes (mit vaterunser, credo und dekalog) auch die vom Salzburger erzbischof Leonhard (1495-1519) erlassene verlautbarung enthält, daß mit dem sprechen des gebetes ein hunderttägiger $a b l a ß$ verbunden sei; nach einer nachricht von 1779 soll auch in der St. Paulskirche von Ortenstein (Graubünden) eine marmortafel mit einem altdeutschen vaterunser und credo gefunden worden sein (ilber 
den überlieferten text s. E. yon Steinmeyers Kleinere althochdeutsche sprachdenkmäler s. 28).

Mit dem nachweis, daß im 15. und 16. jh. dentsche vaterunser (usw.) in kirchen auf steintafeln als ablaßgébete angebracht waren, ergibt sich eine plausible erklärungsmöglichkeit für die herkunft der vorlage von Ad., die die copie einer solchen steininschrift sein könnte: ein frommer laie oder geistlicher mag sie als ablaßgebet für sich copiert haben. Aber auch die wahl eines ahd. textes für die steininschrift läßt sich nun wenigstens hypothetisch einigermaßen begreiflich machen: bei inschriften ist neigung $\mathrm{zu}$ archaisierung nichts unerhörtes, und ein hoher geistlicher auftraggeber des 15.-16. (17.) jh.'s, der antiquarische interessen hatte, mag die alte sprachform als etwas besonders ehrwürdiges, von der alltagssprache sich feierlicher abhebendes empfunden haben, was nicht notwendig peinliche akribie der schreibung in sich schließt; die compilation zweier texte freilich bleibt nach wie vor unaufgeklärt.

Trifft diese erklärung zu, dann würden als vorstufen von Ad. anzusetzen sein: 1 . die hss. der ahd. texte; 2 . die handschriftliche compilation $\mathrm{C}$ als vorlage für den steinmetz; 3. die steininschrift; 4. die copie davon auf dem blatt (s. nr. 3), das Hetterich (5.) abschrieb. Die 'gothische' schrift von H.'s vorlage (s. nr. 3) würde sich (worauf E. v. Steinmeyer mich hinwies) als nachbildung der fraktur der steininschrift besonders einleuchtend erklären, und mit ihr auch die verlesungsmöglichkeit so vieler buchstaben. Den ausfall der zweiten bitte wird man eher dem copisten (4.), als der stufe 2 oder 3 zuschreiben dürfen; auch $H$. scheidet bei der sonstigen genauigkeit seiner abschrift aus. Da nach dieser hypothese Hetterichs abschrift mindestens die fünfte stufe der ahd. texte darstellt (beim $\mathrm{N}$-text, da $\mathrm{N}^{2}$ selbst schon eine späte abschrift war, sogar die sechste), wird natürlich die in nr. 11-13 versuchte aufteilung der abweichungen auf die vorstufen in einzelnen punkten unsicherer; insbesonders wird man hún zweifeln können, ob die verlesungen nicht (insgesamt oder zum teil) schon dem steinmetz (3.) und dem copisten (4.) zur last fallen, eine möglichkeit, der durch hypothetische aufstellung einer mittelstufe $M$ bereits oben rechnung getragen wurde. 
Die datierten zeugnisse für inschriftliche ablaßgebete stammen aus dem 15. und 16. jh. und geben damit auch einen fingerzeig für das wahrscheinliche alter der inschrift, aus der Ad., wenn die hypothese zutrifft, stammt.1) Da es nicht unmöglich ist, dab die vermutete inschrift noch- erhalten sein könnte, wäre es erfreulich, wenn sich die aufmerksamkeit von ortsforschern dem vorkommen solcher vaterunserinschriften in kirchen zuwendete; vielleicht taucht dabei einmal auch der Adelsberger text auf.

1) Sollte die compilation erst im 17. jh. erfolgt sein, so hätte in der zweiten hälfte des 17. jh.'s (bis 1690) die möglichkeit für einen geistlichen des erzstiftes Mainz bestanden, die hs. W in Mainz einzusehen, wo sie um diese zeit sich interimistisch befand, s. von Heinemanns vorwort zu seinem katalog der Weißenburger handschriften (hinweis E. v. Steinmeyers).
WÜRZBURG 1917.
O. L. JIRICZEK. 